\title{
Wetting Dynamics on Solvophilic, Soft, Porous, and Responsive Surfaces - Supporting Information
}

\author{
Sai Ankit Etha, ${ }^{1}$ Parth Rakesh Desai, ${ }^{1}$ Harnoor Singh Sachar, ${ }^{1}$ and Siddhartha Das ${ }^{1 *}$ \\ ${ }^{1}$ Department of Mechanical Engineering, University of Maryland, College Park, MD 20742 \\ *Email: $\underline{\text { sidd@umd.edu }}$
}




\section{S.1. MD Simulation Snapshots}

The MD simulation snapshots showing the coupled dynamics of the polymeric liquid drop and the grafted polymer molecules for the grafting density of $\sigma_{\mathrm{g}}=0.31 / \sigma^{2}$ have been provided in Figs. S1. 
(a) $t=0$
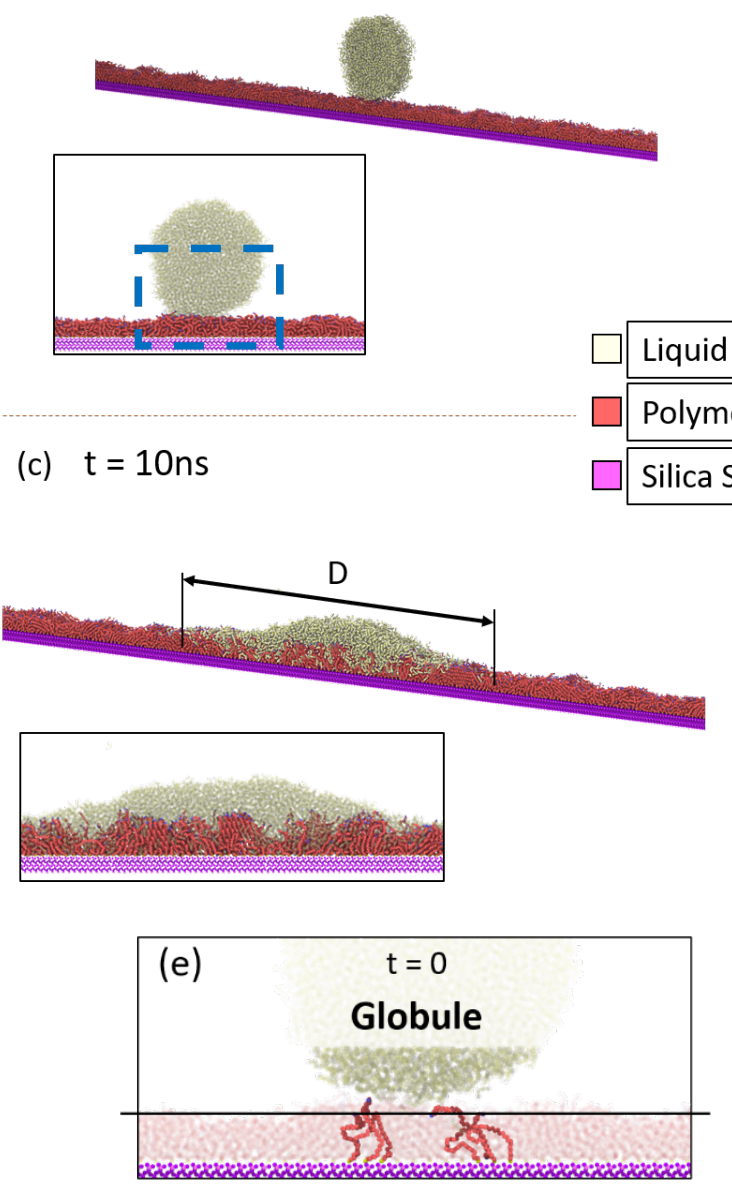

(g)

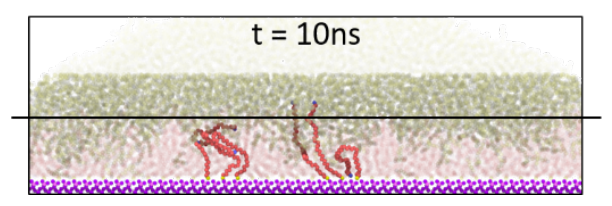

(b) $t=1 n s$
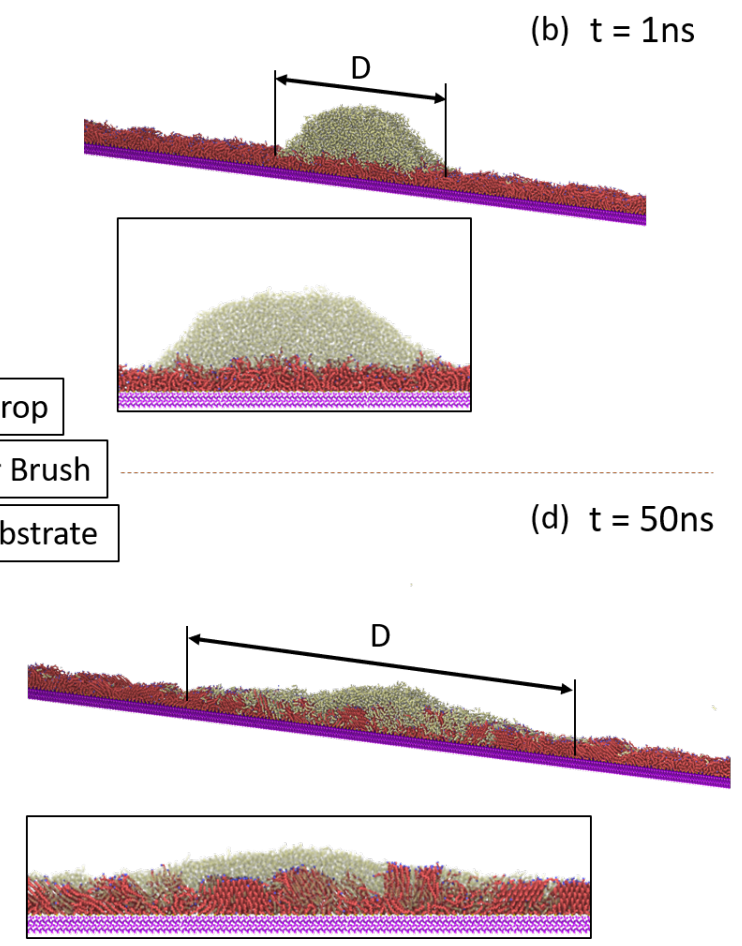

(f)

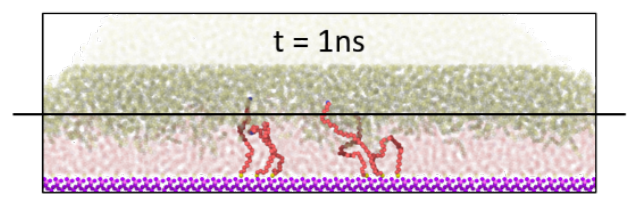

(h)

Brush

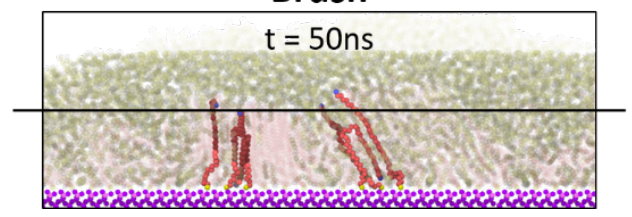

Figure S1. (a-d) MD simulation snapshots showing the interaction of the polymeric liquid drop with the layer of grafted polymer molecules (grafting density $\sigma_{\mathrm{g}}=0.31 / \sigma^{2}$ ) at different time instants (identified on top of the snapshots). For these figures, the interaction is shown by depicting the entire length of the layer of the grafted polymer molecules. In the inset of these subfigures, we highlight a smaller section of the layer of the grafted polymer molecules providing a more detailed view of the solvent-polymer-molecule interface. The simultaneous spreading and wicking of the 
liquid molecules (inside the layer of the grafted polymer molecules) become apparent from these snapshots. In subfigures (a-d), D represents the contact diameter. (e-g) Zoomed images showcasing the behavior of the liquid molecules and the grafted polymer molecules corresponding to region highlighted in (a). These snapshots show that as the liquid penetrates more and more into the polymer layer (the extent of this penetration is represented by noting the location of the liquid front with respect to the black line that provides the instantaneous average height of the grafted polymer molecules), the polymer molecules swell and stretch out to take the form of brushes (the fact that they attain brush-like configuration is established in Fig. 6 in the main paper). For a clearer visualization, we do not show all the polymer molecules along a given length of the solid surface; rather we show only a selected few. 


\section{S.2. Calculation of the Contact Radius (r), Dynamic Contact Angle ( $\theta$ ), Average Time- dependent height of the Grafted Polymer Layer, and Wicking Fraction}

We hereby provide the details for the calculation of the different quantities provided in the main paper. First, we calculate the contact radius $(r)$ of the drop as it spreads. For that purpose, we first divide the system into two-dimensional bins of size $5 \AA \times 5 \AA$ in the vertical plane and filter out bins with insignificant number of liquid molecules, based on their number density (less than $10 \%$ of the maximum number density), at every time instant. This is done so as to obtain the actual shape of the drop and to remove molecules that may have drifted away. Other bin sizes between $3 \AA-7 \AA$ were also tested and we obtain a contact radius variation of less than $2 \%$. Also, the cutoff of $10 \%$ of the maximum number density was chosen, because there is a sharp fall in the number density within the bins beyond this cutoff. This implies that for the bins neighboring the drop surface, the number density falls sharply below the cutoff chosen here (10\%), so that the adjacent bins have of the order of $1 \%$ of the maximum number density. Once this is done, we simply calculate the contact diameter by taking the difference of the minimum and maximum locations of the drop "three-phase" contact line in the x-direction, near the substrate. Of course, after sufficient time, the drop stops spreading and we obtain an equilibrium contact radius. Figure S2 provides a schematic view of the binning procedure and a depiction of the estimated contact diameter. 


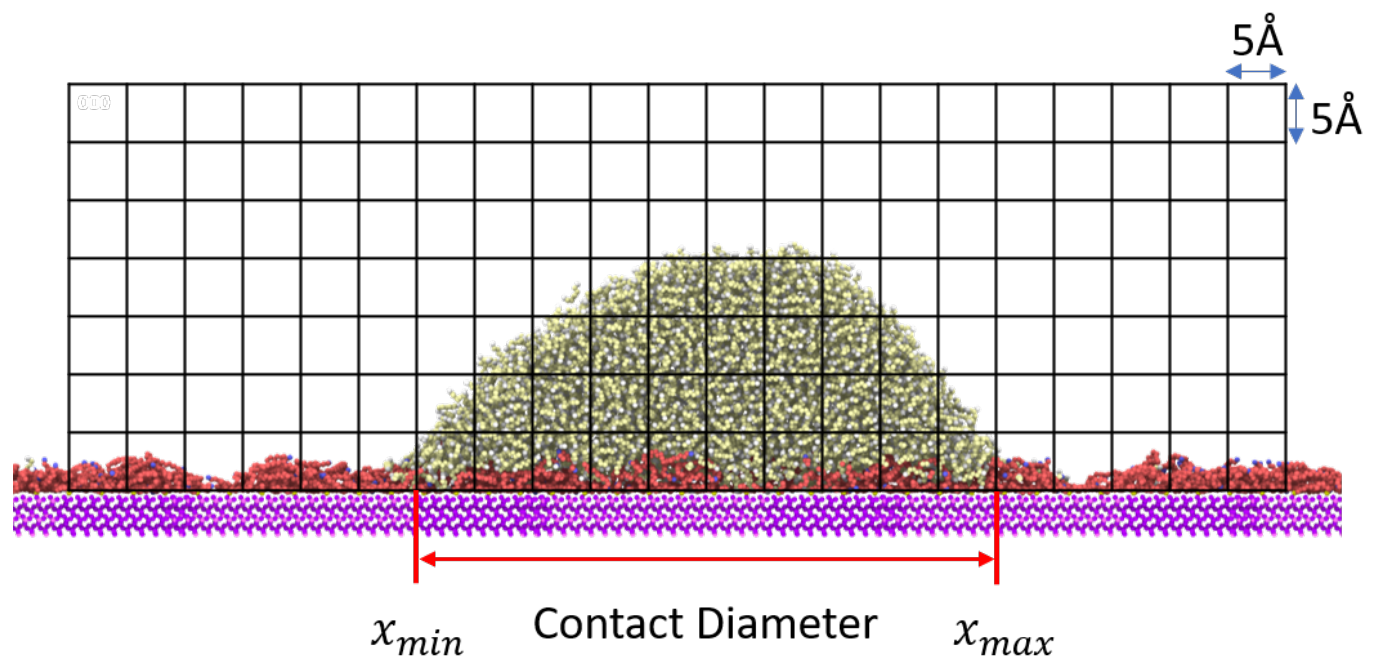

Figure S2. Schematic of the binning procedure to calculate the contact radius of the drop. Each $2 \mathrm{D}$ bin has dimensions of $5 \AA \times 5 \AA$. The red arrow depicts the contact diameter, twice of the contact radius.

Following this, we evaluate the dynamic contact angle $(\theta)$ of the drop, based on a similar approach by Yuan ${ }^{1}$. We divide the liquid molecules of the drop into $3 \AA$ sized bins in the vertical direction (z), upto the maximum height of the drop and note the maximum $\left(x_{\max }\right)$ and minimum $\left(x_{\min }\right) x$ locations of the drop molecules in each of these bins. The drop is defined using the approach employed for the contact radius estimation. Two lines are then fit through each set of the $\mathrm{x}_{\max }$ and $\mathrm{x}_{\min }$ values from 5 adjacent bins nearest to the substrate, respectively, and the slope of these lines are evaluated to determine the contact angle. The slope of the line-fit through the $\mathrm{x}_{\max }$ values would have a negative slope for acute contact angles; therefore, care is taken to compute the contact angle correctly for such a case. Since, there are two contact lines corresponding to the two sides of the 2D drop, we compute the average of the two as the contact angle of the drop at any instant. This process is repeated for the entire simulation time. Figure S3 provides a schematic view of the 
binning procedure, the fitted lines through the lateral locations, and a depiction of the estimated contact angle.

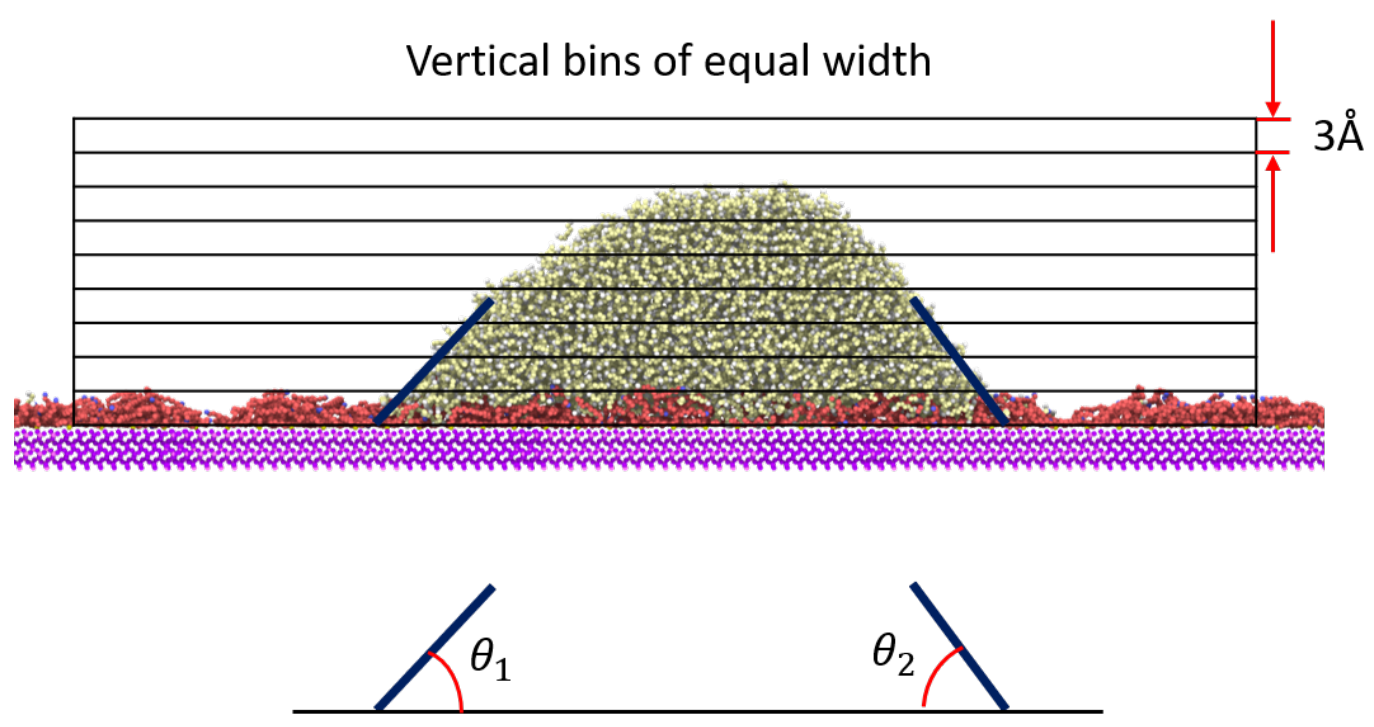

Figure S3. Schematic of the binning procedure to calculate the contact angle of the drop, with bins of width $3 \AA$. The blue lines depict the linear fit through the minimum and maximum lateral locations from the 5 bins nearest to the substrate. The contact angles for the two sides of the drop are represented by $\theta_{1}$ and $\theta_{2}$.

Next, we quantify the height of the grafted polymer layer as it stretches out in the presence of a favorable environment (octane). In Figure 4(a) in the main paper, we have depicted a region that is $x_{w k}=10-\mathrm{nm}$ wide and localized below the drop and around the drop center: in that region, we quantify the dynamics of the polymer chains, as they stretch from a globule-like state to that of a brush, by quantifying the time-dependent average grafted polymer height in that region. We 
employ a trivial approach to compute this quantity: we average the individual heights of each of the polymer chains that are grafted in the chosen section at every time instant. We repeat this procedure for the whole simulation time.

Finally, we analyze the wicking behavior of the liquid molecules as they imbibe into the grafted polymer layer. To quantify this, we determine the number density of drop molecules that have imbibed into the substrate (any molecule that is within the grafted polymer layer, i.e., it has a vertical location less than the average polymer chain height, obtained from the previous result) at any time instant, within the region identified in Fig. 4(a) (a region that is $x_{w k}=10-\mathrm{nm}$ wide and localized below the drop and around the drop center). The result is normalized with respect to the maximum number density of imbibed liquid molecules (equilibrium number density inside the polymer layer) and is defined as the wicking fraction in this work. 
Fig. S4 shows the spreading of the droplet on the substrate grafted with the polymer chain (grafting density $\mathrm{xxx}$ ) without the polymers being visible. This allows us to understand the drop spreading more clearly. Fig. S4(c) depicts the equilibrium structure of the drop, and although the contact angle is quite small (only a few degrees), the drop configuration is still preserved (and we do not encounter a liquid film).

(a)

(b)

$$
\mathrm{t}=10 \mathrm{~ns}
$$

$$
\mathrm{t}=2 \mathrm{~ns}
$$

(c)

$$
\mathrm{t}=50 \mathrm{~ns}
$$

Figure S4: MD simulation snapshots confirming the spreading dynamics and attainment of drop equilibrium on the substrate grafted with the polymer chain (grafting density $\sigma_{\mathrm{g}}=0.085 / \sigma^{2}$ ) without the polymers being visible. The time value corresponding to each snapshot has been provided on the right-hand corner of that snapshot. 


\section{S.5. Autocorrelation Function for the Average Brush Height}

In Fig. S5, we provide the autocorrelation function for the average brush height for different grafting density values.

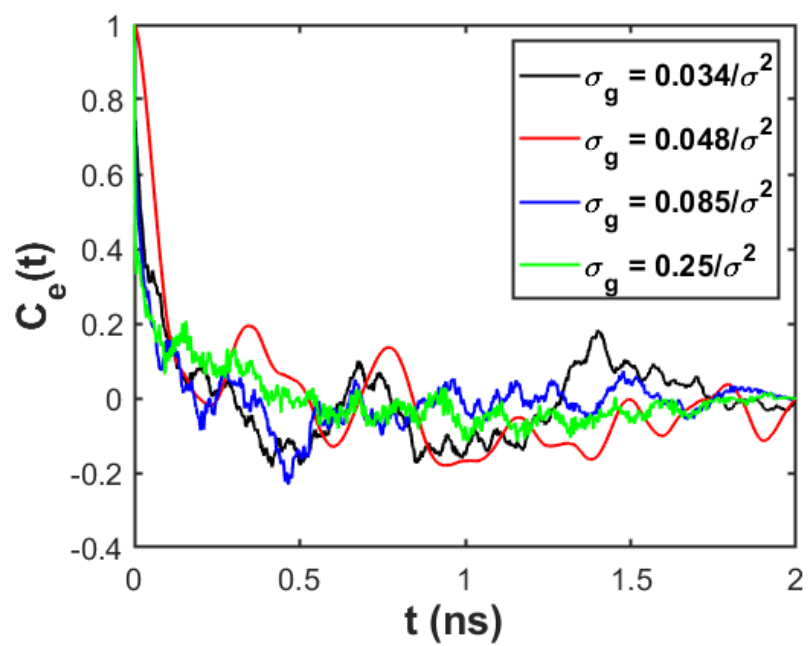

Figure S5: Variation of the autocorrelation function for the average brush height for different grafting density values. 


\section{References}

1. Yuan, C. A Molecular Dynamics Study of the Spreading of Nano-Droplets. Master's $\begin{array}{llll}\text { Thesis, } & \text { KTH } & \text { Stockholm, } & \text { Sweeden }\end{array}$ http://kiosk.nada.kth.se/utbildning/grukth/exjobb/rapportlistor/2010/rapporter10/yuan_ch angming 10144.pdf 